\title{
Understanding diffusion in nanoporous materials
}

\author{
E. Beerdsen and B. Smit
}

University of Amsterdam, Van 't Hoff Institute for Molecular Sciences, Nieuwe Achtergracht 166,1018 WV Amsterdam, The Netherlands. E-mail: beerdsen@science.uva.nl

\begin{abstract}
Can we predict the diffusion behavior of molecules in confinement by looking at the match between the molecule and the structure of the confinement? This question has proven difficult to answer for many decades. As a case study, we use methane and a simple model of ellipsoids to arrive at a molecular picture that allows us to make a classification of pore topologies and to explain their diffusion behavior as a function of loading. Our model is surprisingly simple: regarding a structure as consisting of interconnected ellipsoids is enough to understand the full loading dependence.
\end{abstract}

\section{INTRODUCTION}

The pores of nanoporous materials have sizes similar to the dimensions of molecules adsorbed in them and therefore impose a tight confinement. Well-studied though these systems are, their diffusion properties remain poorly understood. Over the last decades, several techniques have been developed to measure or compute the diffusion in these systems. Although it is now often possible to determine the diffusion as a function of adsorbate loading rather accurately, a proper understanding of diffusion behavior is still lacking.

In an elaborate study, comparing the diffusion of four gases in four zeolite topologies, Skoulidas and Sholl found widely varying diffusion trends, showing the potential of tuning diffusion for industrial processes by adjusting the loading[1,2]. Despite the importance for many applications, conventional methods cannot explain when and why, for a given system, the diffusion will increase, decrease, or remain constant as a function of loading. In this work, we make use of a new Transition-State Theory method in combination with a very simple model based on ellipsoids, to present a fundamental understanding of the loading dependence, and analyze the molecular factors causing the observed behavior. The methodology applied here to methane in various zeolite structures, can be used for any combination of adsorbent and adsorbate, including cation-containing structures [3].

\section{METHODS}

Diffusion can be considered an activated process, in which the particle hops from one cage to the next, and the actual crossing time is negligible compared to the time a particle spends inside the cage. One can exploit the large separation in time scales using rare-event simulation techniques. We consider a system which can be in two stable states, $A$ and $B$, with a dividing free energy barrier between them. We define a reaction coordinate $q$, that indicates the 
progress of the diffusion event from cage $A$ to cage $B$, as the Cartesian coordinate along the axis parallel to the line connecting the center points of $A$ and $B$. The location of the dividing barrier is denoted by $q^{*}$. In the Bennett-Chandler approach [4-6], one computes the hopping rate over the barrier in two steps. First, the relative probability $P\left(q^{*}\right)$ is computed to find a particle on top of the barrier, given that it is in state $A$, and subsequently the averaged velocity at the top of the barrier $\sqrt{k_{B} T / 2 \pi m}$ (assuming that the particle velocities follow a MaxwellBoltzmann distribution) and the probability $\kappa$ that the system ends up in state $B$.

The transmission rate $k_{A \rightarrow B}$ from cage $A$ to cage $B$ is then given by

$$
\begin{aligned}
& k_{A \rightarrow B}=\kappa \times \sqrt{\frac{k_{B} T}{2 \pi m}} \times P\left(q^{*}\right) \\
& P\left(q^{*}\right)=\frac{e^{-\beta F\left(q^{*}\right)}}{\int_{\text {cageA }} e^{-\beta F(q)} d q}
\end{aligned}
$$

where $\beta=1 / k_{B} T, k_{B}$ is the Boltzmann constant, $T$ the temperature, $m$ the mass involved in the reaction coordinate, and $F(q)$ the free energy as a function of $q$. In first order approximation, TST assumes that all particles that reach the barrier with a velocity towards $B$ do end up in $B$, i.e. $\kappa=1$.

In dynamically corrected TST (dcTST), the transmission coefficient $\kappa$ corrects for recrossing events, i.e. it corrects for trajectories which cross the transition state from $A$ but fail to end up in $B$. In general, the reaction coordinate $q$ is a function of the configuration of the whole system, i.e. $q=q\left(\boldsymbol{r}_{1}, \ldots, \boldsymbol{r}_{N}\right)$. However, we can choose $q$ as the position of one of the atoms of the diffusing molecules[7]. This choice of order parameter underestimates the free energy of the true transition state, but the dynamical correction $\kappa$ is the exact correction compensating our choice of reaction coordinate[5]. The recrossings are fast events and can be computed using MD as the fraction of particles coming from the initial state $A$ that successfully reaches the final state $B$ out of those that cross the dividing surface at $t=0$. The transmission coefficient reaches a clear plateau value as a function of time, indicating all short time scale recrossings have been eliminated.

In the limit of infinite dilution there are no inter-particle correlations and the particles perform a random walk on a lattice spanned by the cage-centers. The transmission rates are then easily converted to self-diffusion coefficients by:

$D_{S}=k_{A \rightarrow B} \lambda^{2}=1 / 6 k \lambda^{2}$

with $\lambda$ the center-to-center lattice distance of the LTA cages (12.2775 $\AA$ ). Because we calculate the hopping rate from $A$ to $B$ in one direction only, $k_{A \rightarrow B}=1 / 6 k$.

We compute the self-diffusion coefficient directly. This is done by computing the hopping rate of a molecule over a typical length-scale $\lambda$ given by the smallest repeating zeolite-structure (i.e. from the center of cage A to the center of cage B, implicitly integrating over all adsorption sites in the cage, irrespective of whether these are well-defined or not). The other particles are regarded as a contribution to the external field exerted on the tagged particle. Since we look at a single tagged particle, the diffusion coefficient can still be computed from the hopping rate by using Eq. 3 at any loading, rendering it unnecessary to perform N-particle kMC simulations. Now, $k_{A} \rightarrow_{B}$ is the effective hopping rate, including all jump correlations and averaged over all orientations and loading fluctuations. The external field is maintained by an MC NVT simulation (fixed total number of particles, volume, and temperature) in the 'background'. By using an MC approach that includes translational, 
orientational, and regrow moves, we automatically average over cage distributions, positions, and orientations of neighboring molecules. To speed up these simulations for longer molecules by several orders of magnitude, these techniques can be combined with the configurational bias Monte Carlo (CBMC) approach [6].

We now discuss the two steps in the computation of the hopping rate using our approach in detail.

The probability $P(q)$

During an NVT-ensemble MC simulation at the required loading we measure the free energy $\mathrm{F}(\mathrm{q})$ by using either the Widom Particle Insertion (WPI) method or Histogram Sampling (HS). WPI uses a probe particle that is inserted at random positions, to measure the energy required for or obtained by insertion of the particle in the system. This energy is mapped onto the reaction coordinate $\mathrm{q}$, using $\beta \mathrm{F}(\mathrm{q})=-\ln \langle\mathrm{e}-\beta \Delta \mathrm{U}\rangle \mathrm{N}$, to produce a free energy profile, where $\langle\mathrm{e}-\beta \Delta \mathrm{U}\rangle \mathrm{N}$ is the average Boltzmann factor over all positions in the slice perpendicular to the reaction coordinate. A "ghost particle" is used as the measuring probe, but the other particles in the system do not feel its presence. In the HS method, a histogram is made of the particle positions, mapped on the reaction coordinate. From the histogram a free energy profile is computed, by using $\beta \mathrm{F}(\mathrm{q})=-\ln \langle\mathrm{P}(\mathrm{q})\rangle$. If needed, statistics can be improved by using importance sampling [6]. At higher loadings, WPI is known to give erroneous results [6]. At loadings as low as 6 methane molecules per cage the WPI method starts to deviate.

The transmission coefficient $\kappa$

We compute the fraction of particles starting on top of the barrier with a velocity towards B that successfully reach cage B. Starting configurations are generated using MC with one particle constrained to the dividing surface and $\mathrm{N}-1$ particles moving around freely. These configurations are then used to compute the ratio in unconstrained NVE-MD simulations, starting with velocities sampled from a Maxwell-Boltzmann distribution at the desired temperature. For this snapshot cage B contains more molecules than cage A, and the barriermolecule has a high probability of recrossing to cage A. In general, the transmission coefficient is much lower than one for chain molecules (even at infinite dilution). Note that during the computation none of the windows are blocked and simultaneous jumps (e.g. from cage $\mathrm{C}$ to cage A, and cage D to cage B) are allowed. As is shown in Fig. 1, our extended dcTST method and MD agree quantitatively.

methane in LTA at $600 \mathrm{~K}-\mathrm{MD}$ and corrected TST

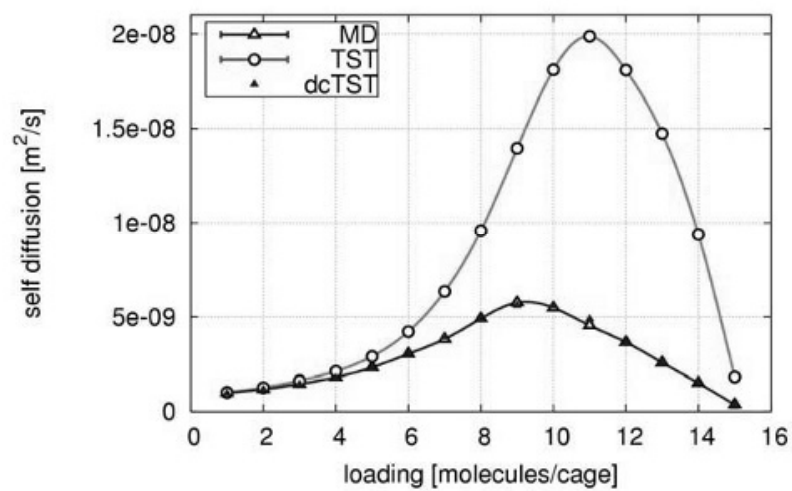

Fig 1: $\mathrm{D}_{\mathrm{S}}$ as a function of loading for methane in LTA, as computed with Molecular Dynamics (MD), Transition-State Theory (TST), and dynamically corrected Transition-State Theory (dcTST). 


\section{RESULTS AND DISCUSSION}

We use a combination of conventional Molecular Dynamics (MD) calculations and our recently proposed dynamically corrected Transition-State Theory (dcTST) method to study the diffusion of methane in a variety of zeolite structures. In addition to diffusion coefficients, this method can yield an explanation of the diffusion behavior in terms of free energy differences. Free energy profiles are computed during an NVT-ensemble MC or MD simulation, in which we compute the probability to find a particle at a particular value of the reaction coordinate $q . D_{S}$ and $D_{C}$ are obtained for methane in 10 different molecular sieve topologies: LTA, CHA, ERI, SAS, AFI, MTW, LTL, MFI, BOG, and BEC; This set represents a wide range of different topologies. We focus on methane, since even for this simple molecule the diffusion behavior is not understood. The results are shown in Fig. 2

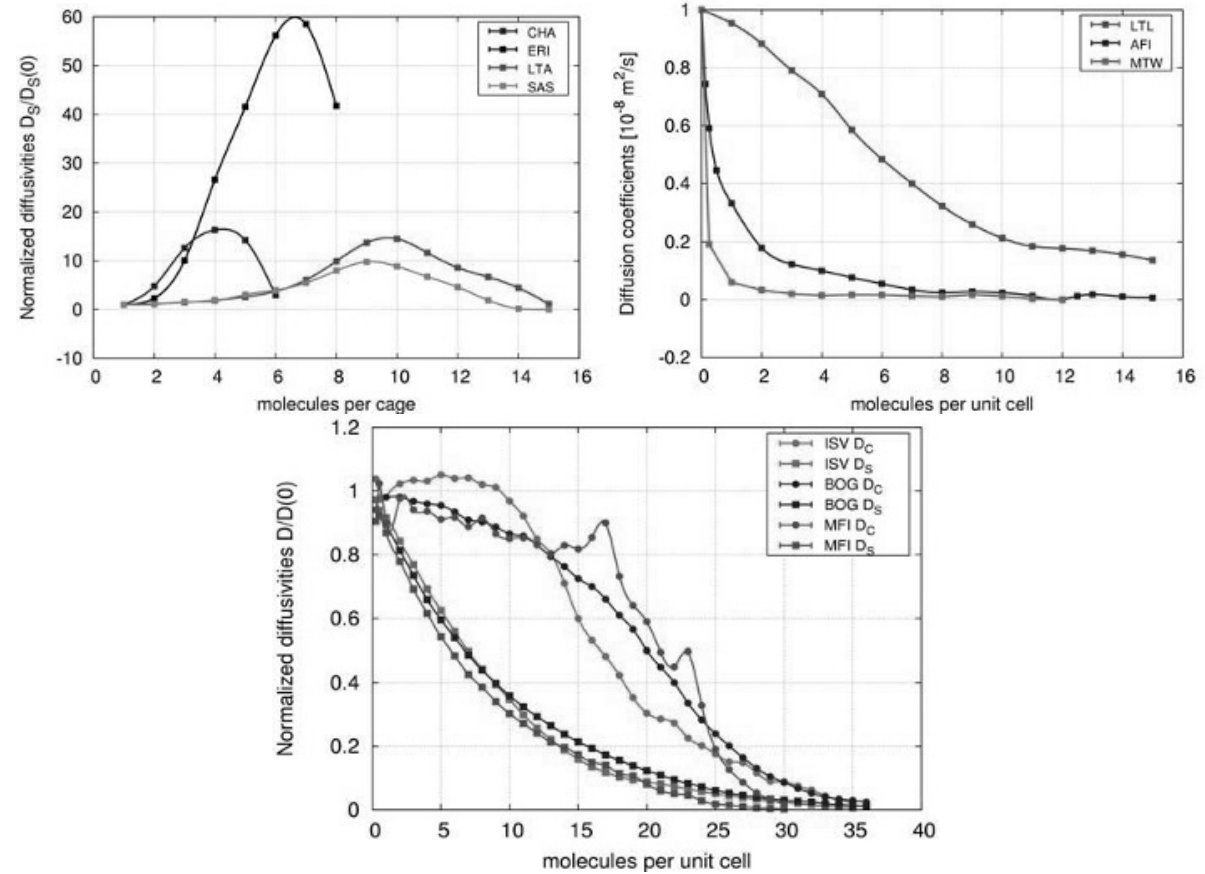

Fig. 2: Normalized $D_{S}$ for cage-type zeolites (top,left), channel-type zeolites (top, right), and intersecting-channel-type zeolites (bottom, along with $\mathrm{D}_{\mathrm{C}}$ ).

We can interpret our results by making use of a very simple concept based on ellipsoids. The molecular sieve's pores or cavities form confinements that can be considered as interconnected ellipsoids. There are three ways to interconnect these ellipsoids (see Fig. 3): aligned in a direction perpendicular to the long axis $a$ (left), aligned along $a$ (middle), or aligned alternatingly (right). The three basic models form confinement types that we refer to as 'cage-type', 'channel-type', and 'intersecting channel-type', respectively, and each of these types gives rise to very distinct diffusion behavior. 

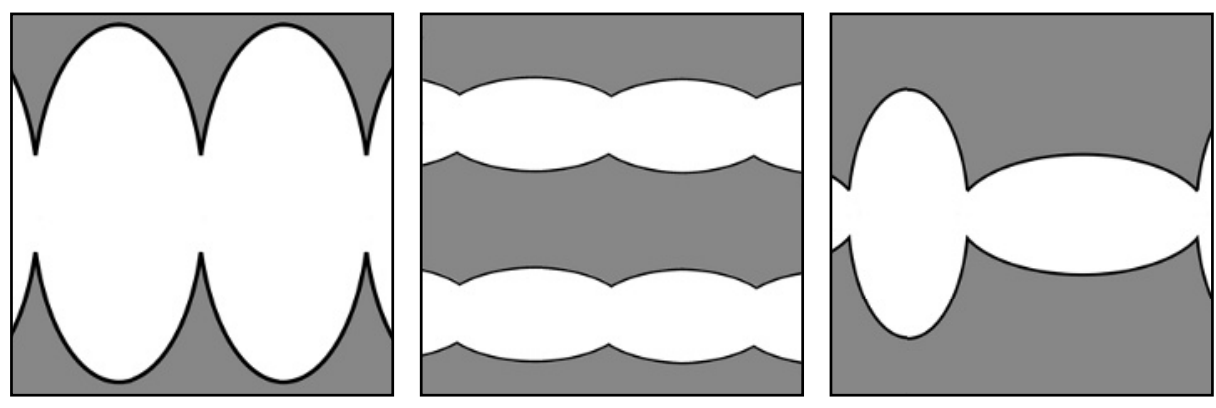

Fig.3: Ellipsoid models used in the explanation of the diffusion behavior in cage-type zeolites (left), channel-type zeolites (middle), and intersecting-channel-type zeolites (right).

The cage-type molecular sieves generally consist of large cages, connected by narrow windows forming large free energy barriers. Adding a new molecule means less interactions with the walls and hence an increase of the free energy of the bottom of the well for methane in LTA-type molecular sieve. The influence of particles at the window region is much smaller, so that as the structure is being filled up, the net free energy barrier decreases, causing an increase in both the self and the transport diffusion coefficient. At very high density, the free energy barrier rapidly increases again at the addition of a molecule, caused by packing and free-volume effects, which causes the diffusion to slow down again.

LTA-, ERI-, CHA-, and SAS-type systems all conform to this scenario for the diffusion of methane. The increase in both self- and corrected diffusion compared to the infinite dilution limit can be a surprising two orders of magnitude. As expected, $D_{C}>D_{S}$ in all cases, due to positively contributing correlations present in $D_{C}$, but not in $D_{S}$.

The second class of confinement consists of channel-type molecular sieves. Upon insertion of new molecules, again the free energy in the interior of the cage rises, but this time the effect on the free energy is even larger at the barriers, mainly due to reduced entropy with respect to the cage regions. As a result, the diffusivity (both $D_{S}$ and $D_{C}$ ) is a decreasing function of loading. The details of the diffusion graph depend on the exact topology of the channels. The smoother the channel (i.e. the wider the windows with respect to the cages), the steeper the decreasing function will be. In channel-type structures, the amount of collective behavior is much higher than in cage-type structures, because the barriers are lower. The difference between $D_{S}$ and $D_{C}$ depends on the window size: the smaller the intersection between ellipsoids, the larger the ratio $D_{C} / D_{S}$.

The third class of confinement is the class of intersecting channel-type structures, of which MFI is the most famous example. Any type of structure with channels running in different directions that mutually intersect, falls into this category. The barriers are formed by the horizontally aligned ellipsoids, creating entropic traps between consecutive vertical ellipsoids. The influence of loading in these systems is complex as it involves effects such as non-simultaneous freezing in vertical and horizontal ellipsoids/channels, due to differences in ellipsoid diameter and length, causing varying degrees of commensurability of the particles with the structure, as a function of loading and direction. Here, like in the case of channeltype molecular sieves, the self diffusion still sharply decreases when the loading is increased, but the corrected diffusivity initially only slightly decreases with density, until packing effects sharply decrease the corrected diffusivity to zero, causing a kink in the diffusion curve at 
intermediate loading. The position of the highest free energy barrier is not the same for every loading. Importantly, the loading at which the corrected diffusion starts its fast decrease corresponds to the loading at which the low-loading highest barrier is overtaken by a barrier at a different position, giving rise to a new diffusion regime.

\section{CONCLUSION}

We have compared the loading-dependent behavior of the self-diffusion and corrected diffusion for methane in ten different zeolite topologies, using a new dynamically corrected Transition-State Theory model. Based on their characteristics, we can divide these topologies into three zeolite groups. Each of the three zeolite groups shows very distinct diffusion behavior as a function of loading. The method employed in this study can be used to make a classification of pore structures for any given adsorbate molecule.

\section{REFERENCES}

[1] A. Skoulidas and D. S. Sholl, J. Phys. Chem. A. 107 (2003) 10132.

[2] A. I. Skoulidas, D. S. Sholl, and R. Krishna, Langmuir 19 (2003) 7977.

[3] E. Beerdsen, B. Smit, and D. Dubbeldam, Phys. Rev. Lett. 93 (2004) 248301.

[4] C. H. Bennett, in Diffusion in Solids: Recent Developments, A. Nowick and J. Burton, ed., Academic Press, New York, 1975, 73.

[5] D. Chandler, J. Chem. Phys. 68 (1978) 2959.

[6] D. Frenkel and B. Smit, Understanding Molecular Simulation, Academic Press, London, 2002, $2^{\text {nd }}$ ed.

[7] D. Dubbeldam, S. Calero, T. L. M. Maesen, and B. Smit, Phys. Rev. Lett. 90 (2003) 245901. 